animals were killed at the end of the experiment. The pathological changes in aortic was observed by HE colouring, masson staining and oil red staining. CRP, MMP-9, ICAM-1 and NF- $\mathrm{KB} / \mathrm{p} 65$ were tested with ELISA, immunochemistry or RT-PCR.

Results Pathological observation showed that thin fibrous cap and big lipid core area was the feature of plaque in the model group. The main components of plaque were macrophages and fat, collagen and actin content of plaque was little, which had showed the pathological characteristics of unstable plaque. We found atherosclerotic lesions of aorta in two drug groups were lighter than in model group $(p<0.05$ or $p<0.01)$. Si Miao Yong An decoction was superior to Simvastatin in increasing fibrous cap thickness and actin content, reducing lipid core area and MMP-9 expression in plaque $(\mathrm{p}<0.01)$. However, there was no significant difference between the two drugs in reducing NF- $\mathrm{KB} / \mathrm{p} 65$ and ICAM-1 mRNA expression ( $\mathrm{p}>0.05)$.

Conclusions Based on these results, we believe that Si Miao Yong An decoction can promotes atherosclerotic plaque stability by fighting against inflammatory, inhibiting matrix degradation and lipid deposition.

\section{e0212 L-CARNITINE TREATMENT IMPROVES SURVIVAL AND EFFECTS OF TRANSPLANTED BONE MARROW MESENCHYMAL STEM CELLS IN POST-INFARCT RATS HEARTS}

doi:10.1136/hrt.2010.208967.212

Peilei Li, Ming Lin, Changsheng Xu. Department of Cardiology, The First Affiliated Hospital, Fujian Medical University, Fuzhou, Fujian, China

Objective To investigate if the L-carnitine can improve the survival rate of transplanted MSCs after myocardial infarction, and enhance therapeutic effect of MSCs.

Methods Five rats were separately obtained, MSCs were isolated and purified by density-gradient centrifugation and adherence method. 60 rats were randomly assigned into five groups: sham operation group $(n=12)$, model group $(n=12)$, LC group $(n=12)$, MSCs group $(n=12)$, and LC+MSCs group $(n=12)$. Rats in the sham operation group received chest open, without ligation of the left coronary artery. In other four groups, the left coronary artery was ligated to establish myocardial infarction models. Following $20 \mathrm{~min}$ of coronary artery ligation, $250 \mu \mathrm{MSCs}\left(2 \times 10^{6}\right.$ cells per animal) were injected into the left ventricular wall of the infarcted hearts $(50 \mu \mathrm{l}$ into1 injected foci) and peri-infarct zone (200 $\mu$ into 4 injected foci) in MSC group and LC+MSCs group, rats in the model group and sham operation group received intramyocardial injection of the same volume of cell-free DMEM. From 3 days prior to MSC transplantation to ended 4 days post-transplantation, rats in the LC group and LC+MSCs were separately administrated with LC $(100 \mathrm{mg} /(\mathrm{kg} \cdot \mathrm{d}))$ intraperitoneally. Rats in the model group, sham operation group and MSCs group separately administrated with PBS $(100 \mathrm{mg} /(\mathrm{kg} \cdot \mathrm{d}))$. The heart function was evaluated by left ventricular ejection fraction, shortening fraction, and the indexes of blood dynamics 4 weeks after transplantation, the survival of MSCs and myocardial fibrosis in myocardial infarction were detected using immunohistochemistry.

Results Compared with the model group, ejection fraction, fractional shortening, the left ventricular end-systolic pressure (LVESP), left ventricular end-diastolic pressure (LVEDP), the maximal rate of isovolumetric contraction ( $+\mathrm{dp} / \mathrm{dtmax},-\mathrm{dp} / \mathrm{dtmax}$ ) and myocardial fibrosis were improved in the group MSCs, group LC and group LC+MSCs $(p<0.05)$; Compared with the MSCs group, the survival of MSCs, ejection fraction, fractional shortening, LVESP, LVEDP, $+\mathrm{dp} / \mathrm{dtmax},-\mathrm{dp} / \mathrm{dtmax}$ and myocardial fibrosis in group LC + MSCs have significantly improved $(p<0.05)$.

Conclusion Cardiac function and myocardial fibrosis can be improved by LC and MSCs in acute myocardial infarction rat models, but the effect is limited. Pretreated with LC, MSCs transplantation will achieve better result for improving the survival of MSCs.

\section{E0213 COMPARISON OF OLMESARTAN MEDOXOMIL AND CANDESARTAN CILEXETIL ON ANTI-INFLAMMATORY EFFECT IN ATHEROSCLEROTIC RATS}

doi:10.1136/hrt.2010.208967.213

${ }^{1}$ Liang Hui, ${ }^{2}$ Chen Huanzhen. ${ }^{1}$ Dalian Friendship Hospital, Liaoning, China; ${ }^{2}$ Department of Cardiology, The First Hospital Affiliated, China

Objective hs-CRP serum level and the CD40 mRNA expression of aorta in atherosclerotic (AS) rats were measured in this experiment To observe the effect of anti-inflammatory on AS rats treated with olmesartan medoxomil, and further to compare the effect between olmesartan medoxomil and candesartan cilexetil.

Methods 40 male Wistar rats were randomly divided into four groups: A control group, B AS model group, C candesartan cilexetiltreated group, D olmesartan medoxomil-treated group's, C and D groups reproduced AS model, C and D group while giving candesartan cilexetil and olmesartan medoxomil to intervene respectively. 1. $2 \mathrm{ml}$ of blood was collected at 0 week and the end of 8 week, then centrifuged and the supernatant was obtained, hs-CRP serum level was detected with enzyme-linked immunosorbent assay (ELISA), to investigate and compare hs-CRP serum level in each group. 2. To separated the thoracic aorta and abdominal aorta of rats quickly, the expression of CD40 of aorta in every group was detected by reverse transcript polymerse chain reaction (RT-PCR), and finally to take pictures and observe with UV detector. Meanwhile with GADPH as an internal reference agent and conclude the relative expression level of CD40 in aortic tissue, to compare the expression of CD40 in rats aortic among the groups.

Results 1 . At 0 week, the levels of serum hs-CRP were similar among the four groups and showed no significant difference ( $p>0.05$ ); At the end of 8 week, the level of serum hs-CRP in AS rats were significantly higher than that in control group rats $(p<0.01)$, among the two treated groups and no treated group, hs-CRP serum level was reduced obviously in two treated groups $(p<0.01)$, furthermore the level of serum hs-CRP in olmesartan medoxomiltreated group was much lower than candesartan cilexetil $(p<0.01)$. 2. The expression level of CD40 in AS rats aorta was obviously higher than control group $(p<0.01)$, but compared with B group, CD40 mRNA expression in aorta of olmesartan medoxomil and candesartan cilexetil-treated rats decresed significantly $(p<0.01)$, the level expression of CD40 in D group was lower than $C$ group and the difference had statistically significant $(p<0.01)$.

Conclusion 1. The level of serum inflammatory likeas hs-CRP was increased in AS rats, and expressed high level of CD40 in AS rats aorta. 2. Olmesartan medoxomil and candesartan cilexetil can reduce AS rats hs-CRP serum level and decrease the expression level of CD40 in aorta. Both of olmesartan medoxomil and candesartan cilexetil have the effect of anti-inflammatory to AS rats. 3. Compared with candesartan cilexetil-treated, the level of serum hs-CRP and the expression of CD40 decrease in olmesartan medoxomil-treated AS rats, therefore olmesartan medoxomil has a stronger anti-inflammatory effects on AS rats. 